\title{
External Electro-Optic Probing of Millimeter-Wave Integrated Circuits
}

J.F. Whitaker and J.A. Valdmanis

University of Michigan

Ann Arbor, Michigan

T.A. Jackson

University of Rochester

Rochester, New York

K.B. Bhasin and R. Romanofsky

Lewis Research Center

Cleveland, Ohio

and

G.A. Mourou

University of Michigan

Ann Arbor, Michigan

Prepared for the

1989 IEEE MTT-S International Microwave Symposium

Long Beach, California, June 13-15, 1989 


\title{
EXTERNAL ELECTRO-OPTIC PROBING OF MILIMETER-WAVE INTEGRATED CIRCUTTS
}

\author{
J.F. Whitaker*, J.A. Valdmanis*, T.A. Jackson ${ }^{*}$, K.B. Bhasin $\dagger$, R. Romanofsky ${ }^{\dagger}$, and \\ G.A. Mourou* \\ * Ultrafast Science Laboratory, Department of Electrical Engineering and Computer Science, \\ University of Michigan, Ann Arbor, MI 48109-2122
}

†† Laboratory for Laser Energetics, University of Rochester, Rochester, NY 14623-1299

$\dagger$ NASA Lewis Research Center, Cleveland, OH 44135

\begin{abstract}
An external, non-contact electro-optic measurement system, designed to operate at the wafer level with conventional wafer probing equipment and without any special circuit preparation, has been developed. Measurements have demonstrated the system's ability to probe continuous and pulsed signals on microwave integrated circuits on arbitrary substrates with excellent spatial resolution. Experimental measurements on a variety of digital and analog circuits, including a GaAs selectively-doped heterostructure transistor prescaler, an NMOS silicon multiplexer, and a GaAs power amplifier MMIC are reported.
\end{abstract}

\section{INTRODUCTION}

The characterization of both devices and circuits in the millimeter-wave range and beyond requires instrumentation possessing unprecedented resolution. The all-electronic network analyzer is essential to the determination of the RF and highfrequency properties of microwave components, but this measurement technique not only has an operating frequency range which is limited to the millimeter-wave regime, but it is also seriously deficient in terms of flexibility. Contacts to a circuit for measurement purposes, such as wire bonds or "Cascade-type" probes, not only may prove to be destructive to the circuit, but they also add the effects of their parasitic circuit parameters to the resulting data, and these effects must be de-embedded to find the actual circuit response. Furthermore, the bandwidth covered by any one set of test fixtures is limited, and in order to test over a broad spectrum at a wide variety of nodes in a millimeter-wave integrated circuit (MMIC), a substantial amount of time for each circuit is required. Thus, it is obvious that a much more flexible network analysis system for testing electrical devices and circuits operating in and above the millimeter-wave regime will soon be required.

\section{EXTERNAL ELECTRO-OPTIC CHARACTERIZATION}

A technique for making extremely broadband measurements of electrical signals which currently exists involves the interaction of the disciplines of optics and electronics: electro-optic sampling (1). An external, non-contact, non-destructive, relatively non-invasive $(2,3)$ variation of this technique can be used to make rapid measurements of electrical quantities, including the S-parameters of a circuit or device. This external electro-optic sampling technique closely approaches the ideal probe for integrated circuits: applicability to circuits fabricated on any substrate; high temporal resolution so that one measurement can cover all frequencies of interest; high spatial resolution for ease of testing small structures; no contact requirements so that probing of internal nodes may be accomplished; low perturbation of signals in the circuit; table-top, room temperature test environment; compatibility with low-temperature testing. This probe, designed to operate at the wafer level with conventional wafer probing equipment and without any special circuit preparation, has been developed and demonstrated. The principle employed is the same as that used in another measurement technique, internal electro-optic probing (4), except that neither an electro-optic substrate nor optical polishing of the substrate are required for external testing.

The probing technique indicated employs an extremely small electro-optic crystal as a proximity electric-field sensor, determining electric potential by measuring the strength of the fringing electric field. This is accomplished when the guided field near the surface of the integrated circuit linearly induces a change in the index of refraction in the electro-optic tip, and a picosecond laser pulse reflecting off the bottom of the crystal resolves the 
speed of this index change. Since the optical probe beam may be focused down to a spot size less than 5 $\mu \mathrm{m}$ in diameter at the bottom of the crystal, excellent spatial resolution is achieved using this testing method. Additionally, no contact to the circuit is necessary, and thus no charge is removed. In this way, very brief electrical transients, with broad, continuous spectra, can be measured in order to determine the input to an MMIC, the reflection from the integrated circuit, and the output of the circuit. Since the electro-optic medium is external to the MMIC, this technique does not rely on any optical properties of the circuit substrate itself, and a wide variety of circuit embodiments may be tested.

The positions of the electro-optic probe beams for two different sampling configurations are shown in Fig. 1(a). The transverse crystal, which would be made of lithium tantalate (LT), is used to measure a fringing e-field perpendicular to the probe beam, while the longitudinal crystal, fabricated of deuterated potassium dihydrogen phosphate $\left(\mathrm{KD}^{*} \mathrm{P}\right)$, is used to measure field components that are parallel to the probe beam (perpendicular to the surface of the electrodes). When the latter tip is used, the most accurate measure of the potential of that electrode carrying the broadband waveform is obtained. Figure 1(b) indicates the relative field strength measured over each of the electrodes of a coplanar waveguide using the two electro-optic probes. The longitudinal probe, which may be reflected off of an electrode rather than a highreflectance coating as shown in Fig. 1(a), is seen in Fig. 1(b) to measure the e-field corresponding to $+V$ only over the extent of the center conductor. The transverse probe tip, however, is used to measure the field normal to the probe beam so that $+V$ is only found over a small range of position directly between the electrodes. The longitudinal tip therefore is able to determine the voltage much more quickly and accurately by sensing the e-field over an arbitrary position of a line, while it is also immune to crosstalk fields from neighboring transmission structures.

The temporal resolution of this technique is fundamentally dictated by the duration of the probing optical pulse, since it acts as the sampling gate in the electro-optic sampling oscilloscope. In our case, measurements were made using 80 -fs long pulses at a wavelength of $630 \mathrm{~nm}$ from a collidingpulse mode-locked dye laser. However, the ultimate frequency response is determined by the first lattice resonance of the electro-optic crystal used as the modulator, which in general occurs in the terahertz regime. In the case of lithium tantalate, the crystal used for transverse electro-optic probing, this resonance occurs in excess of $6 \mathrm{THz}$, and this limits the time response of the probe to approximately 300 fs. The Fourier transform measurement bandwidth of this type of probe is thus greater than one terahertz.

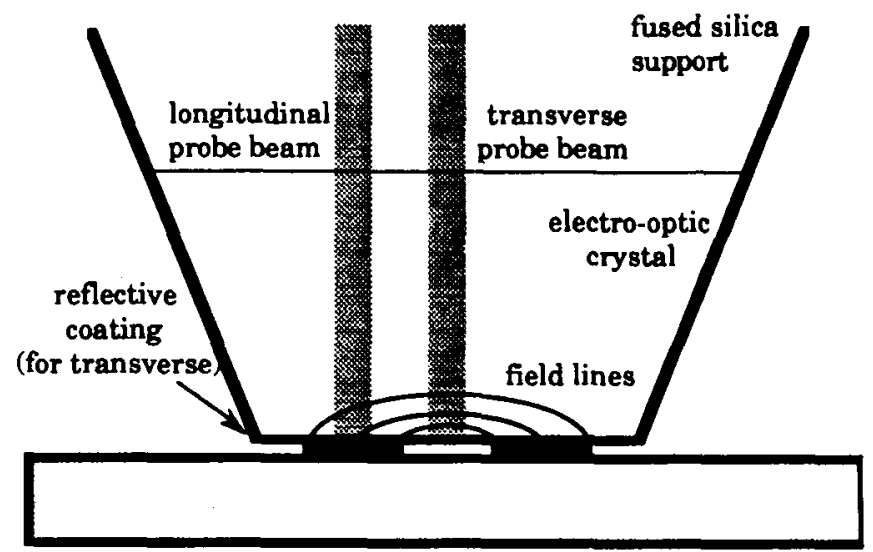

IC substrate

Fig. 1. (a) The external electro-optic probe tip, showing two different modulator configurations, transverse and longitudinal. With a transverse electro-optic crystal, the optical beam direction is perpendicular to the electric field lines fringing above an integrated circuit. With a longitudinal crystal, the optical beam and electric field lines are parallel. The probe beams represent a train of short laser pulses used to time-resolve an electrical signal.

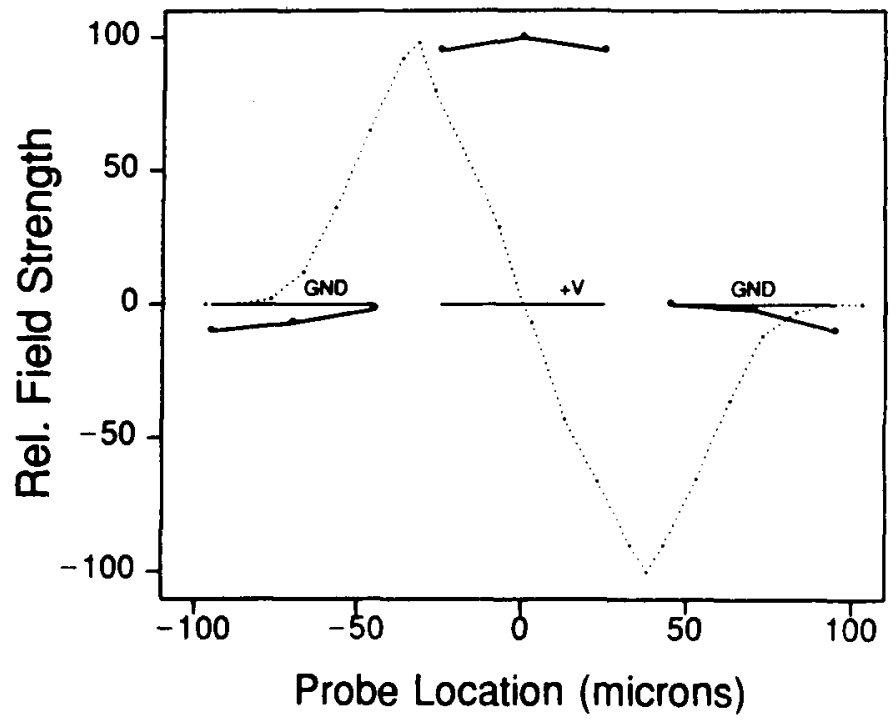

Fig. 1. (b) Field strength - which may be translated to voltage - as measured versus position on a coplanar waveguide using external electro-optic sampling. The solid line indicates the field strength measured with the longitudinal $\mathrm{KD} * \mathrm{P}$ crystal, while the dotted line indicates that measured using the transverse lithium tantalate crystal. 


\section{RESULTS}

The external electro-optic testing technique has been applied to several integrated circuits in order to prove its utility. The first of these to be discussed is a digital circuit, a GaAs selectively-doped heterostructure transistor prescaler. In this case, waveforms on a transmission line with $4-\mu \mathrm{m}$ features at the output of the input buffer of the circuit were measured. The buffer consisted of two banks of three inverter stages, each generating clock and complementary clock output signals. Each inverter consisted of two transistors, one for switching and one for an active load. Measurements were made at this internal node of the circuit on small lines in order to show that signals could be probed with excellent spatial resolution at points inaccessible to conventional "Cascade-type" probes. Figure 2 demonstrates the buffer output when a $1.0-\mathrm{GHz}$ continuous signal is input to the circuit, and the dc bias is varied from above the switching threshold of the inverter chain (where $V_{\text {th }}$ for each inverter is $0.4-0.5 \mathrm{~V}$ ) to below threshold.

A 1.0-GHz clock waveform was also measured on-chip across a 4- $\mu \mathrm{m}$ space between an input clock line and an adjacent power supply line in a highspeed NMOS silicon 12-bit multiplexer. This sine wave, shown in Fig. 3, is an example of a noncontact, electro-optic measurement of a signal in an integrated circuit having a non-electro-optic

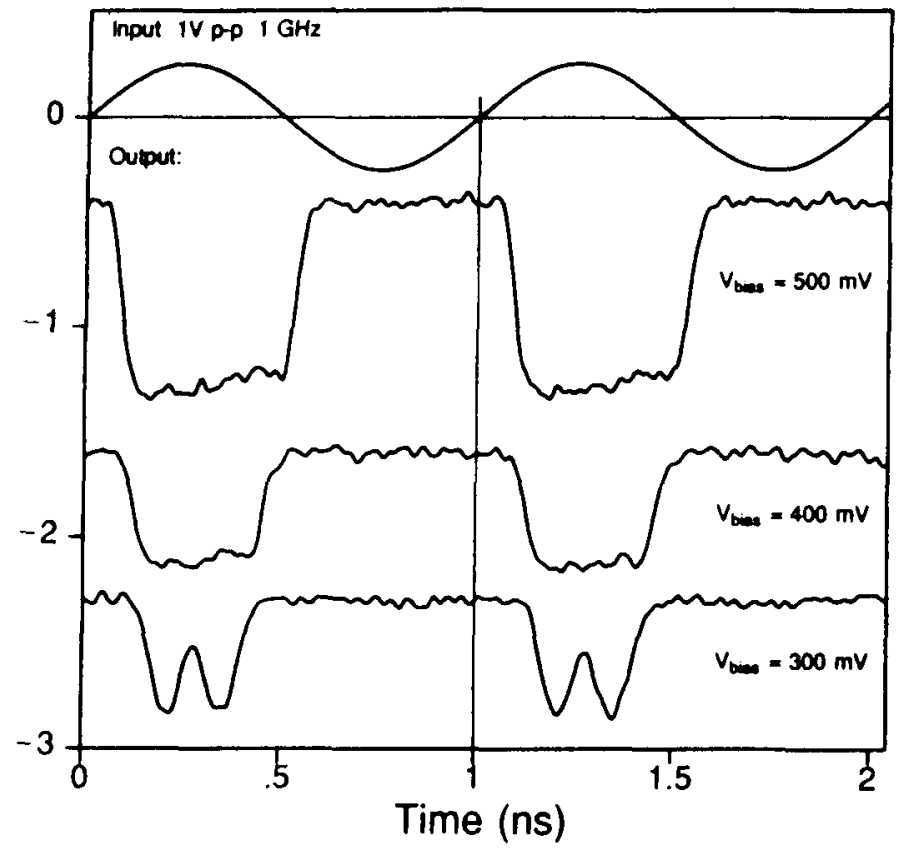

Fig. 2. Output of the inverting input buffer of a GaAs selectively doped heterostructure transistor (SDHT) prescaler integrated circuit with a $1-\mathrm{GHz}$ input signal.

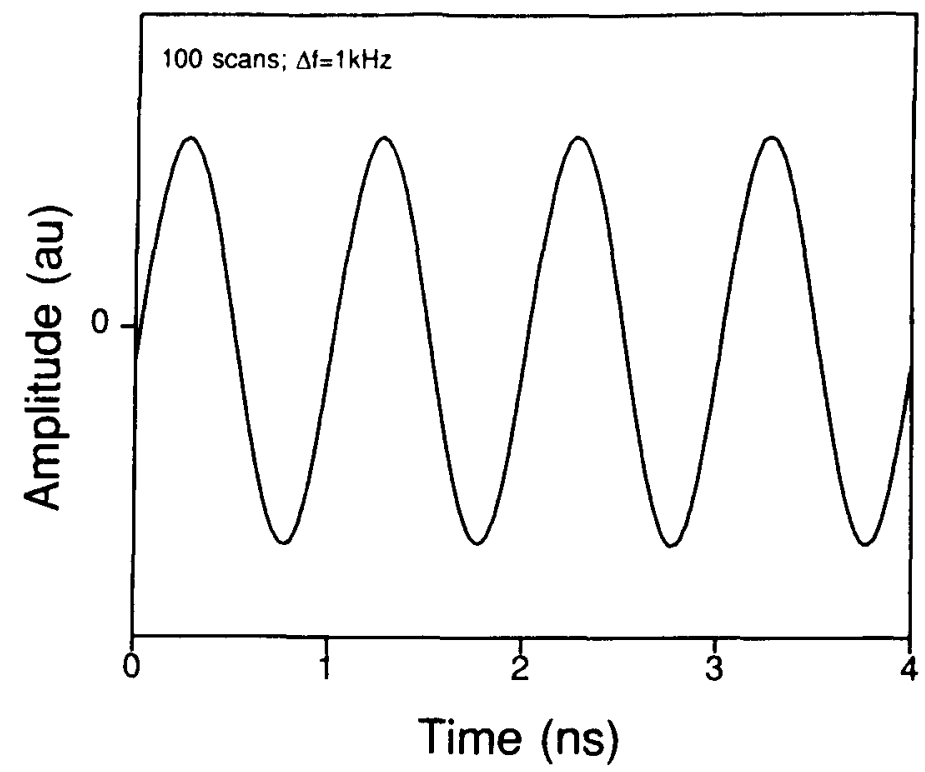

Fig. 3. 1-GHz on-chip clock signal on a silicon 12-bit multiplexer integrated circuit.

substrate material. (i.e., not GaAs, as used in ref. 4.) The clock signal was approximately $200 \mathrm{mV}$ peak-to-peak, and it was generated by phase-locked microwave driver circuitry and scanned at a $1-\mathrm{kHz}$ rate. In this particular case, 100 scans have been averaged in $400 \mathrm{~ms}$ and an excellent signal-to-noise ratio has been obtained.

Measurements were also made on several millimeter-wave, monolithic microwave integrated circuits (MMIC). In this case the signal to be measured was not a synthesized sinusoidal waveform, but rather it originated at the output of a photoconductive switch excited by another 80 -fs laser pulse split off from the optical probe beam. Electrical signals with extremely short rise times can be generated in this fashion (5). These step impulses were introduced to the first gate of $0.4-\mu \mathrm{m}$ gate-length MESFETs in Texas Instruments 100$\mathrm{mW}$, single and double stage power amplifier MMICs. The output electrical signals were then probed above the microstrip transmission lines at the outputs of the amplifier stages. Figure 4 displays the Fourier transforms of the measured time-domain waveforms for three different drain voltages in the single stage MMIC. Similar waveforms were obtained at the outputs of the first and second stages of the two-stage amplifier, with the latter exhibiting greater amplitude. Gain over a narrow band centered at $32.5 \mathrm{GHz}$ was observed. In this case an optical rail provided the variable delay between the probe beam and the excitation beam for the photoconductive switch. Each scan of the delay rail provides data points that are displayed versus time on a signal averager; a scan normally takes seconds to complete. 


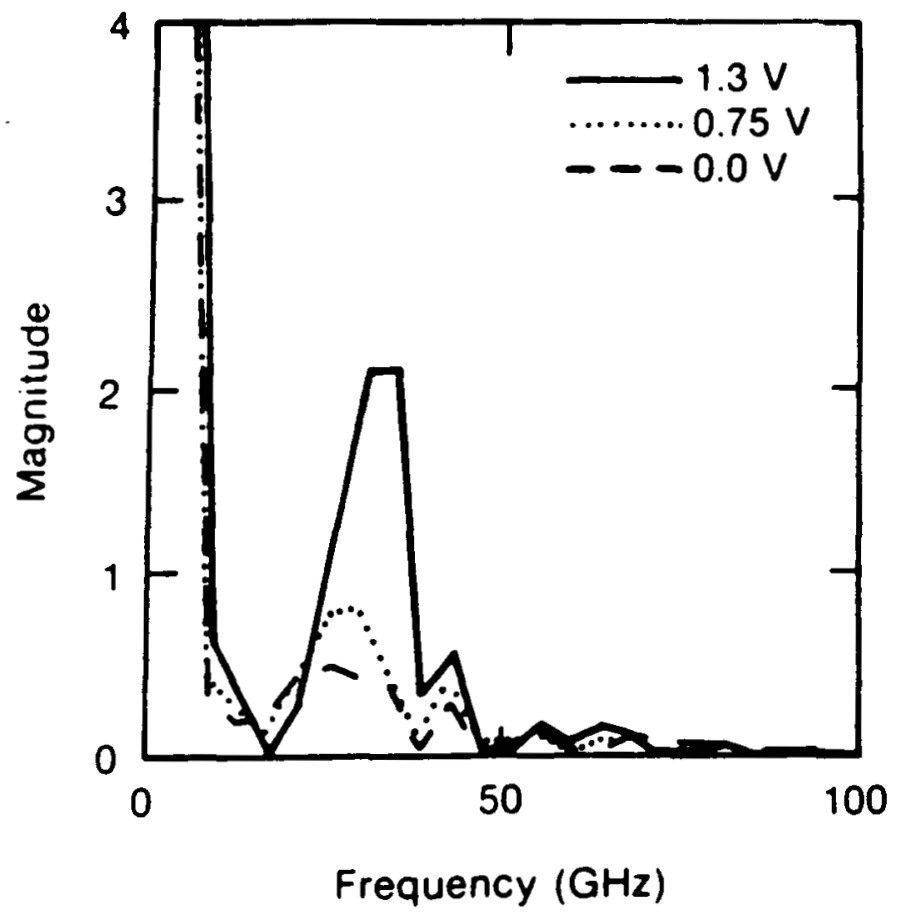

Fig. 4. Spectra of waveforms sampled at the output of a narrow-band MESFET single-stage amplifier MMIC for three different values of drain bias (up to saturation).

\section{CONCLUSION}

We have demonstrated that the technique of external electro-optic sampling can be useful for probing microwave signals on integrated circuits fabricated on arbitrary substrates, having various open-boundary electrode configurations with fewmicron dimensions. In addition, it has been shown that the electro-optic probe tip can measure voltage values on an integrated circuit chip, even in areas where physical contact made by a test instrument would be inappropriate. Measurements in excess of $100 \mathrm{GHz}$ are easily within the capabilities of the electro-optic sampling technique, as future work will prove.

\section{ACKNOWLEDGEMENT}

Support for this work was provided by the National Aeronautics and Space Administration under contract no. NCC3-130. This work was also supported by the Laser Fusion Feasibility Project at the Laboratory for Laser Energetics which has the following sponsors: Empire State Electric Energy Research Corporation, New York State Energy Research and Development Authority, Ontario Hydro, and the University of Rochester. Additional support was provided by the United States Air Force Office of Scientific Research under contract F4962087-C-0016 to the Ultrafast Optical Electronics Center at the Laboratory for Laser Energetics of the University of Rochester. Such support does not imply endorsement of the contract by any of the above parties.

\section{REFERENCES}

1. J.A. Valdmanis, G.A. Mourou, and C.W. Gabel, Appl. Phys. Lett. 41, 211 (1982).

2. J. Nees and G. Mourou, Electron. Lett. 22, 918 (1986).

3. J.A. Valdmanis and S.S. Pei, Picosecond Optics and Electronics II, F.J. Leonberger, C.H. Lee, F. Capasso, and H. Morkoc, Eds. (Berlin: Springer-Verlag, 1987)

4. K.J. Weingarten, M.J.W. Rodwell, and D.M. Bloom, IEEE J. Quantum Electron. 24, 198, (1988).

5. G. Mourou, K. Meyer, J. Whitaker, M. Pessot, R. Grondin, and C. Caruso, Picosecond Optics and Electronics II, F.J. Leonberger, C.H. Lee, F. Capasso, and H. Morkoc, Eds. (Berlin: Springer-Verlag, 1987) 


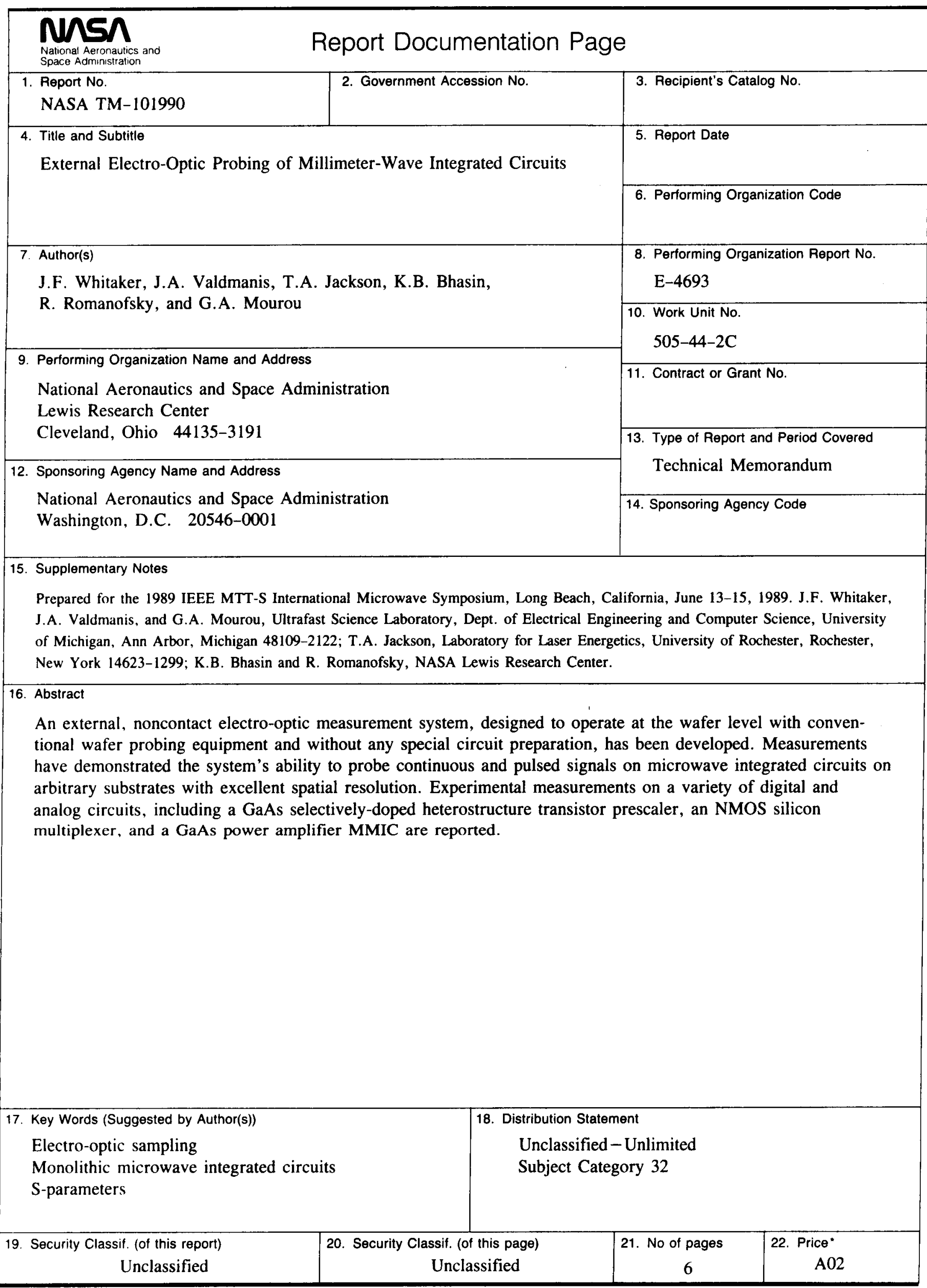

